\title{
Morphological variation in Paspalum nicorae Parodi accessions, a promising forage
}

\author{
Camila Aparecida de Oliveira dos Reis'; Miguel Dall'Agnol²; Carlos Nabinger²; Maria \\ Teresa Schifino-Wittmann ${ }^{2 *}$ \\ ${ }^{1}$ UFRGS/FA - Programa de Pós-Graduação em Zootecnia. \\ ${ }^{2}$ UFRGS/FA - Depto. de Plantas Forrageiras e Agrometeorologia, C.P. 15100 - 91501-970 - Porto Alegre, RS - \\ Brasil. \\ *Corresponding author <mtschif@ufrgs.br>
}

\begin{abstract}
Paspalum nicorae Parodi is a perennial, apomictic tetraploid forage species, native in the state of Rio Grande do Sul, Brazil, with grazing tolerance and adapted to sandy soils and moderate droughts. As a first step to future breeding, a morphological characterization regarding leaf hairiness, sheath, leaf and central venation color, growing habit, plant height, raceme number and length, and leaf length and width was performed in 53 accessions from Rio Grande do Sul that are part of a Paspalum species breeding program. $35.84 \%$ of the accessions had completely hairy leaves, $73.58 \%$ had green sheath, $54.71 \%$ whitish central venation, $50.94 \%$ decumbent habit. Leaf color was estimated by two methods, color chart and colorimeter. The accessions were classified as $76.92 \%$ green, $13.45 \%$ greenish yellow and $9.62 \%$ as grayish green by the first, and $59.62 \%$ grayish, $32.69 \%$ grayish yellow, $5.77 \%$ yellow and $1.92 \%$ dark grayish by the second method. Raceme length ranged from 9.40 to $1.30 \mathrm{~cm}$, number of racemes from one to six $(48.73 \%$ of the accessions had four racemes), leaf length and width from 36.13 to $13.06 \mathrm{~cm}$ and 0.67 to $0.36 \mathrm{~cm}$, respectively, and plant height from 115.70 to $29.00 \mathrm{~cm}$. Therefore, a large morphological variation among accessions was detected, with no relation to geographical location but indicating a high potential use in plant breeding programs.
\end{abstract}

Key words: apomixis, forage grass, morphology, plant breeding

\section{Variação morfológica em acessos de Paspalum nicorae Parodi, forrageira promissora}

\begin{abstract}
RESUMO: Paspalum nicorae Parodi é uma forrageira perene, apomítica, tetraplóide, nativa no Rio Grande do Sul, com tolerância ao pastejo e adaptada a solos arenosos e a secas moderadas. Como passo inicial para o melhoramento, uma caracterização morfológica considerando pilosidade da folha, cor da bainha, da folha e da nervura central, hábito de crescimento, altura da planta, número e comprimento de racemos, comprimento e largura da folha foi realizada em 53 acessos coletados no Rio Grande do Sul, Brasil, que são parte de um programa de melhoramento de espécies de Paspalum. Os resultados mostraram que $35,84 \%$ dos acessos tinham folhas completamente pilosas, $73,58 \%$ tinham bainha verde, $54,71 \%$ venação central esbranquiçada e $50,94 \%$ hábito decumbente. A cor da folha foi estimada por dois métodos, cartela de cores e colorímetro Os acessos foram classificados como 76,92\% verdes, $13,45 \%$ amarelo esverdeados e $9,62 \%$ como verde acinzentados pelo primeiro, e 59,62\% acinzentados, 32,69\% amarelo acinzentados, 5,77\% amarelos e 1,92\% cinza escuros pelo segundo método. O comprimento do racemo variou de 9,40 a $1,30 \mathrm{~cm}$, o número de racemos de um a seis (sendo que 48,73\% dos acessos tinham quatro racemos), o comprimento e a largura da folha de 36,13 a 13,06 $\mathrm{cm}$ e 0,67 a $0,36 \mathrm{~cm}$, respectivamente e a altura da planta de 115,70 a $29,00 \mathrm{~cm}$. Portanto, grande variação morfológica foi detectada entre os acessos, sem relação com localização geográfica, mas indicando alta potencialidade de utilização em programas de melhoramento.

Palavras-chave: apomixia, gramínea forrageira, morfologia, melhoramento
\end{abstract}

\section{Introduction}

Many of the 400 species of genus Paspalum L., most of them native to tropical and sub-tropical Americas (Moraes-Fernandes et al., 1968) are important forages, as the well studied $P$. notatum Flügge (Pedreira and Pedreira, 2006), due to quality, productivity and tolerance to environmental stresses (Valls, 2000). About 75\% of the described Paspalum species occur in Brazil, in a wide range of ecological conditions as part of several plant communities (Barreto, 1974). Apomixis and sexual reproduction, both occurring in this genus, are generally related to the polyploid and diploid levels, respectively (Quarín and Norrmann, 1990). Paspalum nicorae Parodi ("grama cinzenta", brunswickgrass), of the Plicatula group, is a perennial species, highly tolerant to grazing (Barreto, 1974), tetraploid with $2 \mathrm{n}=4 \mathrm{x}=40$ chromosomes (Burson and Bennett, 1970; Moraes-Fernandes et al., 1974; Pagliarini et al., 2001; Reis et al., 2008) and has a pseudogamous apomictic mode of reproduction 
(Burson and Bennet 1970). The importance of P. nicorae is due not only to its good quality and forage yield potential but also because it has a high potential to be used for the recovery and conservation of degraded soil, as mentioned by several authors (Burson and Bennett, 1970; Prestes et al., 1976; Pizzarro, 2000; Boldrini, 2006; Dall'Agnol et al., 2006). In Rio Grande do Sul, P. nicorae is generally found in sandy soils, indicating a potential to tolerate drought and low-fertility soils, and has a high response to fertilization (Nabinger and Dall'Agnol, 2008) and in such areas it could be a promising forage. However, detailed morphological and agronomic evaluations of a high number of accessions are needed to assess the real potential of the species.

The objective of this study was to investigate morphological variation for several characteristics in a collection of 53 P. nicorae accessions that are part of a breeding project including several Paspalum species. This same collection has been cytogenetically analyzed by Reis et al. (2008).

\section{Material and Methods}

The 53 original individual plants were collected in 17 sites in Rio Grande do Sul, Southern Brazil (Table 1). Plants with different characteristics were chosen by the collectors in each site in order to collect a representative sample of the local existing variability. Plants were transferred to pots filled with soil and kept as part of a germplasm collection. The accessions (each of the 53 plants represents an accession) are identified in the collection by the species' name, the collector's number and collection site (Table 1) and numbered for working purposes. Later, as the original plants were well grown, they were vegetatively replicated. After the plants had flowered, their taxonomic status was confirmed. All the accessions are tetraploid $(2 \mathrm{n}=40$ chromosomes) as determined by Reis et al. (2008).

The following morphological characters were analyzed: leaf hairiness (HAI), leaf color (CCLCOL by the color chart and COLLCOL by the colorimeter methods, as explained below) sheath color (SCOL), central venation color (CVCOL), growing habit (HAB), plant height (PHEI), raceme number (RNUM), raceme length (RLEN), and leaf length (LLEN) and leaf width (LWID). For four of the characteristics not all of the 53 accessions were examined: RNUM (39 accessions), CCLCOL and COLLCOL (52 accessions each), LLEN and LWID (50 accessions).

For leaf length (LLEN) and leaf width (LWID) evaluation, marked tillers were used. For each accession three tillers (representing the replications) were marked and all the completely expanded leaves were measured weekly for nine weeks (from March $3^{\text {rd }}$ to April 27 ${ }^{\text {th }}$, 2007). Leaf hairiness (HAI), was estimated on the upper side of the leaf blade, on a 1 to 5 scale: 1 (no hairs); 2 (25\% of the blade with hairs); $3(50 \%) ; 4(75 \%)$ and 5 (100\%). Leaf color by the color chart method (CCLCOL) was determined using three color groups of the R.S.H
Color Chart of the Royal Horticultural Society of London: green group (chart sub-groups 1 to 5), yellow green group (chart sub-groups 6 to 8) and grayish green group (chart sub-groups 9 to 10). Leaf color by the colorimeter method (COLLCOL) was determined with a MINOLTA CR-300 colorimeter (Konika Minolta, USA) and using the CIE (Comission International de 1'Éclairage) L*a*b System: the values were divided in four groups, 1- dark grayish (values 1 -10), 2- light grayish (11-20), 3- grayish yellow (21-30) and 4- yellowish (3140). Sheath color (SCOL) and central venation color (CVCOL) were estimated visually, and considered to be green (1) or violet (2) and whitish (1) or greenish (2), respectively. Growing habit (HAB) was classified as erect (1) or decumbent (2). Raceme number (RNUM) and length (RELN) were determined during the peak of flowering in totally expanded inflorescences. Plant height (PHEI) was determined by measuring the plant from the soil to the highest leaf, once a week, from February $8^{\text {th }}$ to April $27^{\text {th }}, 2007$, after a preliminary cut (January $25^{\text {th }}$, 2007) to standardize initial height. Leaf length and width (LLEN and LWID) were determined at the end of the evaluation period, by measuring all the leaves of each of the three tillers per accession.

Data were analyzed by a descriptive statistics (such as averages, standard deviations etc.).

\section{Results and Discussion}

The present work is the first one to present a detailed analysis of morphological variation for several qualitative and quantitative characteristics of a large number of $P$. nicorae accessions (Tables 2, 3, 4 and 5). Regarding leaf hairiness, $16.98 \%$ of the accessions had completely glabrous leaves and $35.84 \%$ completely hairy leaves (Table 2). Therefore, most of the P. nicorae accessions examined were hairy. Barreto (1974) described the species as having hairy or glabrous leaves and Burkart (1969) refers to lightly hairy to subglabrous leaves, seldom very hairy. Boldrini et al. (2005) also mentioned glabrous and hairy biotypes. The present work is the first one to describe variation in degrees of leaf hairiness.

Most of the accessions, $76.92 \%$, were classified as green, $13.45 \%$ as greenish yellow and $9.62 \%$ as grayish green by the color chart. The colorimeter method distinguished four groups: $59.62 \%$ of the accessions were light grayish, $32.69 \%$ grayish yellow, $5.77 \%$ yellowish and $1.92 \%$ dark grayish (Table 2). Boldrini et al. (2005) refer to $P$. nicorae biotypes with greenish or grayish leaves. The colorimetry method has been efficiently used to characterize, for example, post-harvest fruits (Callegaro et al., 2002) and soil color (Botelho et al., 2006). However, the color chart method is more practical to examine plants directly at the field and does not demand laboratory procedures. Most of the accessions, $73.58 \%$, had green and $26.41 \%$ violet leaf-sheaths (Table 2). Barreto (1974) reported green and violet leaf sheaths in Paspalum species of the Plicatula group, but did not use this character when describing $P$. nicorae. Color of 
Table 1 - List of the 53 accessions of P. nicorae analyzed.

\begin{tabular}{|c|c|c|c|}
\hline Accession & Collector $^{\mathrm{a}}$ & Collection site & Geographical coordinates \\
\hline 2 & $\mathrm{MD}, \mathrm{CN} \mathrm{s} / \mathrm{n}$ & Cristal & Lat $-31^{\circ} 00^{\prime} 00^{\prime \prime}$; Long $-52^{\circ} 03^{\prime} 00^{\prime \prime}$ \\
\hline $2 \mathrm{~A}$ & $\mathrm{MD}, \mathrm{CN} \mathrm{s} / \mathrm{n}$ & & \\
\hline 3 & $\mathrm{MD}, \mathrm{CN} \mathrm{s} / \mathrm{n}$ & São Lourenço & 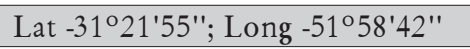 \\
\hline 4 & $\mathrm{MD}, \mathrm{CN} \mathrm{s} / \mathrm{n}$ & Capão do Leão & Lat $-31^{\circ} 45^{\prime} 46^{\prime \prime} ;$ Long -52²9'02" \\
\hline $4 \mathrm{~A}$ & $\mathrm{MD}, \mathrm{CN} s / \mathrm{n}$ & & \\
\hline 5 & $\mathrm{MD}, \mathrm{CN} \mathrm{s} / \mathrm{n}$ & & \\
\hline $6 \mathrm{~A}$ & $\mathrm{MD}, \mathrm{CN} \mathrm{s} / \mathrm{n}$ & Pinheiro Machado & 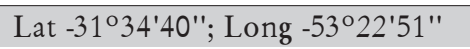 \\
\hline $6 \mathrm{~B}$ & $\mathrm{MD}, \mathrm{CN} s / \mathrm{n}$ & & \\
\hline $8 \mathrm{~A}$ & $\mathrm{MD}, \mathrm{CN} \mathrm{s} / \mathrm{n}$ & & \\
\hline $8 \mathrm{~B}$ & $\mathrm{MD}, \mathrm{CN} \mathrm{s} / \mathrm{n}$ & & \\
\hline 9 & $\mathrm{MD}, \mathrm{CN} \mathrm{s} / \mathrm{n}$ & Pinheiro Machado/Candiota & Lat $-31^{\circ} 33^{\prime} 28^{\prime \prime}$; Long $-53^{\circ} 40^{\prime} 22^{\prime \prime}$ \\
\hline $9 \mathrm{~A}$ & $\mathrm{MD}, \mathrm{CN} \mathrm{s} / \mathrm{n}$ & & \\
\hline 10 & $\mathrm{MD}, \mathrm{CN} \mathrm{s} / \mathrm{n}$ & Hulha Negra/Candiota & 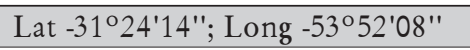 \\
\hline $11 \mathrm{~A}$ & $\mathrm{MD}, \mathrm{CN} \mathrm{s} / \mathrm{n}$ & Bagé & 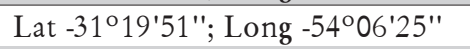 \\
\hline $11 \mathrm{~B}$ & $\mathrm{MD}, \mathrm{CN} \mathrm{s} / \mathrm{n}$ & & \\
\hline $12 \mathrm{~A}$ & $\mathrm{MD}, \mathrm{CN} \mathrm{s} / \mathrm{n}$ & Dom Pedrito & 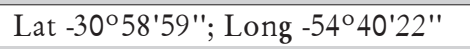 \\
\hline 12B & $\mathrm{MD}, \mathrm{CN} s / \mathrm{n}$ & & \\
\hline $13 \mathrm{~A}$ & $\mathrm{MD}, \mathrm{CN} s / \mathrm{n}$ & & \\
\hline $13 \mathrm{~B}$ & $\mathrm{MD}, \mathrm{CN} \mathrm{s} / \mathrm{n}$ & & \\
\hline 14 & $\mathrm{MD}, \mathrm{CN} \mathrm{s} / \mathrm{n}$ & Dom Pedrito/Santana do Livramento & 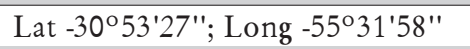 \\
\hline $14 \mathrm{~A}$ & $\mathrm{MD}, \mathrm{CN} \mathrm{s} / \mathrm{n}$ & & \\
\hline 17 & $\mathrm{MD}, \mathrm{CN} \mathrm{s} / \mathrm{n}$ & Santana do Livramento & 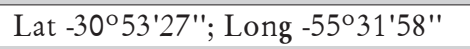 \\
\hline 18 & $\mathrm{MD}, \mathrm{CN} s / \mathrm{n}$ & & \\
\hline 19 & $\mathrm{MD}, \mathrm{CN} s / \mathrm{n}$ & & \\
\hline $20 \mathrm{~B}$ & $\mathrm{MD}, \mathrm{CN} \mathrm{s} / \mathrm{n}$ & & \\
\hline $20 \mathrm{C}$ & $\mathrm{MD}, \mathrm{CN} \mathrm{s} / \mathrm{n}$ & & \\
\hline $26 \mathrm{~A}$ & $\mathrm{MD}, \mathrm{CN} \mathrm{s} / \mathrm{n}$ & Alegrete & Lat $-29^{\circ} 47^{\prime} 02^{\prime \prime} ;$ Long $-55^{\circ} 47^{\prime} 28^{\prime \prime}$ \\
\hline $26 \mathrm{C}$ & $\mathrm{MD}, \mathrm{CN} \mathrm{s} / \mathrm{n}$ & & \\
\hline $26 \mathrm{D}$ & $\mathrm{MD}, \mathrm{CN} s / \mathrm{n}$ & & \\
\hline $26 \mathrm{~F}$ & $\mathrm{MD}, \mathrm{CN} s / \mathrm{n}$ & & \\
\hline $27 \mathrm{~A}$ & $\mathrm{MD}, \mathrm{CN} \mathrm{s} / \mathrm{n}$ & & \\
\hline $27 \mathrm{~B}$ & $\mathrm{MD}, \mathrm{CN} \mathrm{s} / \mathrm{n}$ & & \\
\hline $28 \mathrm{~A}$ & $\mathrm{MD}, \mathrm{CN} \mathrm{s} / \mathrm{n}$ & Rosário do Sul & Lat $-30^{\circ} 15^{\prime} 28^{\prime \prime} ;$ Long $-54^{\circ} 54^{\prime} 50^{\prime \prime}$ \\
\hline $28 \mathrm{~B}$ & $\mathrm{MD}, \mathrm{CN} s / \mathrm{n}$ & & \\
\hline $28 \mathrm{C}$ & $\mathrm{MD}, \mathrm{CN} \mathrm{s} / \mathrm{n}$ & & \\
\hline $28 \mathrm{D}$ & $\mathrm{MD}, \mathrm{CN} s / \mathrm{n}$ & & \\
\hline $28 \mathrm{E}$ & $\mathrm{MD}, \mathrm{CN} \mathrm{s} / \mathrm{n}$ & & \\
\hline 29 & $\mathrm{MD}, \mathrm{CN} \mathrm{s} / \mathrm{n}$ & & \\
\hline $30 \mathrm{~A}$ & $\mathrm{MD}, \mathrm{CN} \mathrm{s} / \mathrm{n}$ & São Gabriel & 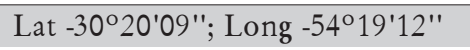 \\
\hline $30 \mathrm{~B}$ & $\mathrm{MD}, \mathrm{CN} \mathrm{s} / \mathrm{n}$ & & \\
\hline $31 \mathrm{~A}$ & $\mathrm{MD}, \mathrm{CN} \mathrm{s} / \mathrm{n}$ & & \\
\hline $31 \mathrm{~B}$ & $\mathrm{MD}, \mathrm{CN} \mathrm{s} / \mathrm{n}$ & & \\
\hline $32 \mathrm{~A}$ & $\mathrm{MD}, \mathrm{CN} \mathrm{s} / \mathrm{n}$ & Santa Margarida do Sul & 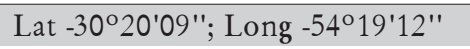 \\
\hline $32 \mathrm{~B}$ & $\mathrm{MD}, \mathrm{CN} \mathrm{s} / \mathrm{n}$ & & \\
\hline $32 \mathrm{C}$ & $\mathrm{MD}, \mathrm{CN} \mathrm{s} / \mathrm{n}$ & & \\
\hline $33 \mathrm{~A}$ & $\mathrm{MD}, \mathrm{CN} \mathrm{s} / \mathrm{n}$ & Vila Nova do Sul & 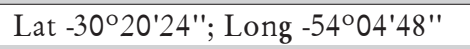 \\
\hline $33 \mathrm{~B}$ & $\mathrm{MD}, \mathrm{CN} s / \mathrm{n}$ & & \\
\hline 34 & $\mathrm{MD}, \mathrm{CN} \mathrm{s} / \mathrm{n}$ & & \\
\hline 35 & $\mathrm{MD}, \mathrm{CN} \mathrm{s} / \mathrm{n}$ & Eldorado do Sul & 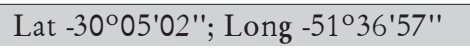 \\
\hline 36 & $\mathrm{MD}, \mathrm{CN} \mathrm{s} / \mathrm{n}$ & & \\
\hline $37 \mathrm{~A}$ & $\mathrm{DN} \mathrm{s} / \mathrm{n}$ & Viamão & 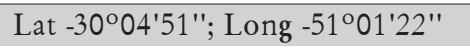 \\
\hline $37 \mathrm{C}$ & $\mathrm{DN} s / \mathrm{n}$ & & \\
\hline $37 \mathrm{D}$ & $\mathrm{DN} s / \mathrm{n}$ & & \\
\hline
\end{tabular}

${ }^{a}$ MD-Miguel Dall'Agnol; CN-Carlos Nabinger; DN-Danilo Menezes Sant'Anna; s/n- not numbered. 
Table 2 - Morphological data in the 53 P. nicorae accessions studied.

\begin{tabular}{|c|c|c|c|c|c|c|c|c|c|c|c|}
\hline Accession & HAI & SCOL & CVCOL & $\mathrm{HAB}$ & CCLCOL & COLLCOL & RNUM & RLEN & PHEI & LLEN & LWID \\
\hline 2 & 2 & 1 & 2 & 1 & 1 & 3 & 4 & 370 & 22.77 & 1306 & 0.43 \\
\hline $2 \mathrm{~A}$ & 4 & 1 & 1 & 1 & 1 & 3 & - & - & 45.14 & 25.35 & 0.50 \\
\hline 3 & 1 & 1 & 1 & 1 & 1 & 2 & 4 & 2.75 & 40.58 & 22.36 & 0.49 \\
\hline 4 & 5 & 2 & 1 & 1 & 1 & 2 & 4 & 3.53 & 57.57 & 34.23 & 0.57 \\
\hline $4 \mathrm{~A}$ & 4 & 1 & 1 & 2 & 1 & 3 & 4 & 3.38 & 44.04 & 28.79 & 0.58 \\
\hline 5 & 1 & 2 & 1 & 2 & 3 & 1 & 3 & 2.60 & 49.63 & 28.04 & 0.39 \\
\hline $6 \mathrm{~A}$ & 1 & 1 & 2 & 2 & 1 & 2 & 4 & 3.38 & 50.91 & 28.11 & 0.46 \\
\hline $6 \mathrm{~B}$ & 5 & 2 & 2 & 2 & 1 & 2 & 5 & 2.98 & 53.57 & 24.99 & 0.51 \\
\hline $8 \mathrm{~A}$ & 2 & 1 & 1 & 1 & 3 & 2 & - & - & 47.89 & 23.42 & 0.44 \\
\hline $8 \mathrm{~B}$ & 2 & 1 & 2 & 1 & 3 & 2 & - & - & 45.51 & 22.19 & 0.36 \\
\hline 9 & 3 & 2 & 1 & 1 & 1 & 3 & 4 & 3.15 & 51.01 & 28.47 & 0.62 \\
\hline $9 \mathrm{~A}$ & 3 & 1 & 1 & 2 & 1 & 2 & 4 & 3.50 & 58.11 & 34.08 & 0.47 \\
\hline 10 & 1 & 1 & 1 & 1 & 3 & 2 & 4 & 2.73 & 52.93 & 28.46 & 0.50 \\
\hline $11 \mathrm{~A}$ & 5 & 1 & 2 & 2 & 2 & 4 & 3 & 3.67 & 54.55 & 32.89 & 0.58 \\
\hline 11B & 4 & 1 & 1 & 2 & 1 & 3 & 4 & 4.68 & 47.62 & 28.18 & 0.62 \\
\hline $12 \mathrm{~A}$ & 5 & 2 & 1 & 2 & 1 & 2 & 4 & 6.98 & 77.20 & 35.38 & 0.67 \\
\hline $12 B$ & 5 & 1 & 1 & 1 & 1 & 2 & 4 & 6.28 & 76.38 & 35.92 & 0.63 \\
\hline $13 \mathrm{~A}$ & 5 & 1 & 2 & 1 & 1 & 2 & 6 & 3.20 & 53.80 & 26.52 & 0.54 \\
\hline $13 \mathrm{~B}$ & 5 & 1 & 1 & 2 & 1 & 2 & 5 & 3.50 & 48.77 & 27.30 & 0.50 \\
\hline 14 & 2 & 1 & 2 & 2 & 1 & 2 & 4 & 4.93 & 38.63 & 20.93 & 0.36 \\
\hline $14 \mathrm{~A}$ & 5 & 1 & 2 & 2 & 1 & 2 & - & - & 45.67 & 21.57 & 0.37 \\
\hline 17 & 1 & 1 & 2 & 2 & 3 & 2 & 2 & 2.35 & 43.83 & 27.85 & 0.54 \\
\hline 18 & 1 & 2 & 2 & 2 & 3 & 2 & 3 & 3.17 & 43.00 & 23.90 & 0.49 \\
\hline 19 & 2 & 1 & 1 & 1 & 1 & 3 & - & - & 78.83 & 28.33 & 0.61 \\
\hline $20 \mathrm{~B}$ & 3 & 2 & 1 & 1 & 1 & 3 & 6 & 3.28 & 56.11 & 31.07 & 0.54 \\
\hline $20 \mathrm{C}$ & 5 & 1 & 1 & 1 & 2 & 2 & - & - & 43.32 & 25.19 & 0.60 \\
\hline $26 \mathrm{~A}$ & 1 & 1 & 1 & 2 & 2 & 2 & - & - & 56.5 & 28.41 & 0.43 \\
\hline $26 \mathrm{C}$ & 2 & 1 & 1 & 1 & 1 & 3 & 5 & 3.26 & 40.07 & 26.01 & 0.38 \\
\hline $26 \mathrm{D}$ & 5 & 1 & 1 & 2 & 1 & 3 & 3 & 4.13 & 75.11 & 36.27 & 0.56 \\
\hline $26 \mathrm{~F}$ & 5 & 1 & 1 & 2 & 1 & 2 & 4 & 3.63 & 51.32 & 26.03 & 0.47 \\
\hline $27 \mathrm{~A}$ & 5 & 2 & 2 & 2 & - & - & 2 & 3.05 & 39.02 & 20.21 & 0.37 \\
\hline $27 \mathrm{~B}$ & 5 & 1 & 2 & 2 & 1 & 3 & 3 & 3.83 & 43.59 & 25.97 & 0.45 \\
\hline $28 \mathrm{~A}$ & 3 & 1 & 1 & 1 & 1 & 2 & 3 & 2.23 & 54.14 & 23.46 & 0.45 \\
\hline $28 \mathrm{~B}$ & 3 & 2 & 1 & 1 & 1 & 2 & 4 & 3.43 & 65.18 & 29.21 & 0.48 \\
\hline $28 \mathrm{C}$ & 3 & 2 & 2 & 1 & 1 & 2 & - & - & 69.28 & 36.13 & 0.56 \\
\hline $28 \mathrm{D}$ & 5 & 1 & 1 & 1 & 1 & 3 & 3 & 4.17 & 59.74 & 29.21 & 0.40 \\
\hline $28 \mathrm{E}$ & 2 & 1 & 1 & 1 & 2 & 2 & 3 & 5.03 & 69.42 & 34.59 & 0.47 \\
\hline 29 & 3 & 1 & 2 & 2 & 2 & 4 & 4 & 3.90 & 43.20 & 24.16 & 0.50 \\
\hline $30 \mathrm{~A}$ & 5 & 1 & 1 & 2 & 1 & 2 & 4 & 3.73 & 54.80 & 32.28 & 0.60 \\
\hline $30 \mathrm{~B}$ & 5 & 1 & 2 & 1 & 1 & 3 & - & - & 39.79 & 29.57 & 0.55 \\
\hline $31 \mathrm{~A}$ & 5 & 1 & 2 & 1 & 1 & 2 & - & - & 37.66 & 24.57 & 0.53 \\
\hline 31B & 2 & 1 & 2 & 1 & 2 & 3 & 4 & 3.68 & 48.89 & 23.35 & 0.44 \\
\hline $32 \mathrm{~A}$ & 3 & 1 & 2 & 1 & 2 & 4 & 4 & 3.98 & 41.22 & 21.24 & 0.49 \\
\hline $32 \mathrm{~B}$ & 3 & 1 & 2 & 1 & 1 & 3 & 5 & 2.92 & 51.41 & 23.75 & 0.50 \\
\hline $32 \mathrm{C}$ & 3 & 1 & 2 & 1 & 2 & 2 & 4 & 2.00 & 46.36 & 28.99 & 0.50 \\
\hline $33 \mathrm{~A}$ & 5 & 2 & 2 & 2 & 1 & 2 & 5 & 3.24 & 45.43 & - & - \\
\hline $33 \mathrm{~B}$ & 4 & 2 & 2 & 1 & 1 & 3 & - & - & 9.55 & - & - \\
\hline 34 & 5 & 1 & 2 & 2 & 1 & 3 & - & - & 34.78 & 22.12 & 0.67 \\
\hline 35 & 2 & 1 & 2 & 1 & 1 & 2 & 5 & 3.16 & 27.77 & 14.65 & 0.43 \\
\hline 36 & 4 & 1 & 1 & 2 & 1 & 3 & - & - & 50.69 & 28.69 & 0.48 \\
\hline $37 \mathrm{~A}$ & 1 & 1 & 1 & 2 & 1 & 2 & - & - & 49.29 & - & - \\
\hline $37 \mathrm{C}$ & 1 & 2 & 1 & 2 & 1 & 2 & 3 & 1.98 & 59.22 & 27.88 & 0.48 \\
\hline $37 \mathrm{D}$ & 2 & 2 & 1 & 2 & 1 & 2 & 3 & 3.54 & 68.88 & 30.81 & 0.46 \\
\hline
\end{tabular}

${ }^{a}$ Average values (see Material and Methods). HAI: leaf hairiness - 1 (absent); 2 (25\%); 3 (50\%); 4 (75\%); 5 (100\%). SCOL: sheath color - 1 (green); 2 (violet). CVCOL: central venation color - 1 (whitish); 2 (greenish). HAB: growing habit - 1 (erect); 2 (decumbent). CCLCOL: leaf color by color chart - 1 (green group); 2 (yellow green group); 3 (grayish green group). COLLCOL: leaf color by colorimeter - 1 (dark grayish); 2 (light grayish); 3 (grayish yellow); 4 (yellowish). RNUM: raceme number. RLEN: raceme length. LLEN: leaf length. LWID: leaf width. 
Table 3 - Descriptive statistics for the seven quantitative characteristics analyzed in P. Nicorae.

\begin{tabular}{lcccccc}
\hline Variable & Variance & Average & Maximum & Minimum & Standard deviation & Number of observations \\
\hline HAI & 2.3606 & 3.2830 & 5 & 1 & 1.5364 & 53 \\
SCOL & 0.1981 & 1.2641 & 2 & 1 & 0.4450 & 53 \\
CVCOL & 0.2525 & 1.4528 & 2 & 1 & 0.5025 & 53 \\
HAB & 0.2547 & 1.5094 & 2 & 1 & 0.5046 & 53 \\
RNUM & 0.8313 & 3.8974 & 6 & 2 & 0.9117 & 39 \\
CCLCOL & 6.8759 & 3.7884 & 10 & 1 & 2.6222 & 52 \\
COLLCOL & 0.405732 & 2.42308 & 4 & 1 & 0.6369 & 52 \\
\hline
\end{tabular}

HAI: leaf hairiness - 1 (absent); 2 (25\%); 3 (50\%); 4 (75\%); 5 (100\%). SCOL: sheath color - 1 (green); 2 (violet). CVCOL: central venation color -1 (whitish); 2 (greenish). HAB: growing habit -1 (erect); 2 (decumbent). RNUM: raceme number. CCLCOL: leaf color by color chart - 1-5 (green group); 6-8 (yellow green group); 9-10 (grayish green group). COLLCOL: leaf color by colorimeter - 1 (dark grayish); 2 (light grayish); 3 (grayish yellow); 4 (yellowish).

the central venation was whitish in $54.71 \%$ and greenish in $45.29 \%$ of the accessions (Table 2).

Regarding growing habit, $50.94 \%$ of the accessions had a decumbent while $49.06 \%$ had an erect habit (Table 2). Raceme number and length varied greatly among the accessions analyzed. Number of racemes ranged from two to six: $48.72 \%$ of the accessions had four, $25.64 \%$ three, $15.38 \%$ five, $5.13 \%$ two and $5.13 \%$ six racemes (Table 2). Burkart (1969), Barreto (1974) and Boldrini et al. (2005) described $P$. nicorae as having from two to five racemes. This is also the first report of six racemes (in two out of 53 of the accessions examined) in the species. Raceme length (average values) ranged from 1.98 to 6.98 $\mathrm{cm}$ (Table 2). Barreto (1974) reported racemes from two to five $\mathrm{cm}$ in $P$. nicorae, while Burkart (1969) referred to 1.5 to $6.5 \mathrm{~cm}$.

Plant height (average values) among accessions ranged from 22.77 to $77.20 \mathrm{~cm}$ (Tables 2 and 4), agreeing with Burkart (1969) who described plants ranging from 20 to $70 \mathrm{~cm}$ high. Average leaf length and width among accessions ranged from 13.06 to $36.27 \mathrm{~cm}$ and from 0.36 to $0.67 \mathrm{~cm}$, respectively (Tables 2 and 5). Burkart (1969) reported leaf width in $P$. nicorae as ranging from 0.4 to $0.5 \mathrm{~cm}$. Leaf length and width divided the accessions basically in three groups: one $(50.00 \%$ of the accessions) with long (more than $20 \mathrm{~cm}$ ) and wide (more than 0.5 $\mathrm{cm})$ leaves, the second ( $46.15 \%$ of the accessions) with long (more than $20 \mathrm{~cm}$ ) and narrow (smaller than $0.5 \mathrm{~cm}$ ) leaves and the third $(3.85 \%$ of the accessions, representing two accessions), with small (less than $20 \mathrm{~cm}$ ) and narrow (smaller than $0.5 \mathrm{~cm}$ ) leaves.

No relations between a specific geographical location and a given characteristic was verified, for the quantitative as well as for the qualitative characteristics (Tables 1 and 2). For example, the different leaf colors and growing habits were found in accessions spread all over the collection area; on the other hand, two accessions (11 A and 11B) collected in the same locality had different leaf colors by both the color chart and the colorimeter methods and other two, also collected at the same place (12A and 12B) had a decumbent (12A) and an erect (12B) habit. Plants belonging to the three groups of leaf length-width were also found all over the collection area.

That variation exists in apomictic species and is well known and reported for several species (Asker and Jerling, 1982). For example, in a germplasm collection of Paspalum notatum, in which morphological, agronomical and molecular variation among different accessions have been reported, Dahmer et al. (2008) found great variability in meiotic configurations among the apomictic tetraploid accessions. Reis et al. (2008), working with the same 53 accessions of $P$. nicorae examined here, observed that, despite the absence of variability in chromosome numbers, the frequencies of different chromosome associations varied among accessions.

The high variation found among accessions, as well as the fact that accessions of the same collection site differed in some characteristics, can be explained by adaptation to the local where plants grow but also by the apomictic mode of reproduction of $P$. nicorae. As apomictic species do not depend on sexual reproduction for survival, any new neutral variation (in the sense that it does not hinder the plant survival or adaptation), that has appeared by mutation or by rare recombination events during the evolutionary history of the population may be maintained. Therefore, plants with different phenotypes may propagate by the apomictic seeds or by vegetative propagation (or even by residual sexuality when it does exist), forming a pool of different clones in a given area (Asker and Jerling, 1982). Many of the characteristics analyzed for $P$. nicorae, as leaf color, sheath and central venation color, among others, are most probably not related to plant adaptation, which could explain the high variation not only among accessions but also between accessions of the same collection site. Furthermore, the existence of sexual types, not yet found for $P$. nicorae but already reported in other Paspalum species (Quarín and Normann, 1990), would allow the continuous formation of new apomictic types through hybridization with true apomictics.

Finally, as a following to the present work, the value of these accessions as forage plants has to be tested in other studies, under field conditions. 
Table 4 - Descriptive statistics for the qualitative characteristic PHEI - plant height (cm) analyzed in P. nicorae.

\begin{tabular}{|c|c|c|c|c|c|}
\hline Accession & Average & Standard deviation & Maximum & Minimum & Variance \\
\hline 2 & 22.7667 & 5.281 & 29.00 & 10.00 & 27.8900 \\
\hline $2 \mathrm{~A}$ & 45.1444 & 7.809 & 51.40 & 29.20 & 60.9878 \\
\hline 3 & 40.5778 & 3.800 & 47.70 & 36.00 & 14.4419 \\
\hline 4 & 57.5667 & 4.715 & 63.20 & 46.80 & 22.2325 \\
\hline $4 \mathrm{~A}$ & 44.0444 & 8.352 & 56.40 & 31.00 & 69.7503 \\
\hline $6 \mathrm{~A}$ & 50.9111 & 2.723 & 53.80 & 47.70 & 7.4161 \\
\hline $6 \mathrm{~B}$ & 53.5567 & 10.978 & 62.50 & 31.00 & 120.5200 \\
\hline $8 \mathrm{~A}$ & 47.8889 & 3.409 & 51.00 & 39.50 & 11.6211 \\
\hline $8 \mathrm{~B}$ & 45.511 & 6.783 & 56.00 & 37.50 & 46.0110 \\
\hline 9 & 51.0111 & 9.119 & 59.80 & 30.00 & 83.1486 \\
\hline $9 \mathrm{~A}$ & 58.1111 & 7.384 & 67.10 & 44.70 & 54.5261 \\
\hline 10 & 52.9333 & 8.202 & 63.30 & 41.90 & 67.2775 \\
\hline $11 \mathrm{~A}$ & 54.5556 & 9.485 & 64.00 & 40.00 & 89.9703 \\
\hline 11B & 47.6222 & 12.515 & 62.30 & 30.00 & 156.6169 \\
\hline $12 \mathrm{~A}$ & 77.2000 & 8.821 & 89.20 & 64.50 & 77.8150 \\
\hline $12 \mathrm{~B}$ & 76.3778 & 6.390 & 87.00 & 67.00 & 40.8369 \\
\hline $13 \mathrm{~A}$ & 53.8000 & 7.651 & 63.60 & 40.20 & 58.5375 \\
\hline $13 B$ & 48.7667 & 9.612 & 58.70 & 28.80 & 92.3950 \\
\hline 14 & 38.6333 & 3.063 & 42.00 & 34.50 & 9.3850 \\
\hline $14 \mathrm{~A}$ & 45.6667 & 8.894 & 57.80 & 34.00 & 79.1100 \\
\hline 17 & 43.8333 & 4.945 & 50.20 & 35.50 & 24.4550 \\
\hline 18 & 43.0000 & 6.061 & 49.00 & 28.50 & 36.7350 \\
\hline 19 & 78.8333 & 16.874 & 11.70 & 54.00 & 284.7400 \\
\hline $20 \mathrm{~B}$ & 56.111 & 3.959 & 60.00 & 47.00 & 15.6711 \\
\hline $20 \mathrm{C}$ & 43.3222 & 5.702 & 50.50 & 34.50 & 32.5094 \\
\hline $26 \mathrm{~A}$ & 56.5000 & 8.685 & 72.60 & 47.00 & 75.4225 \\
\hline $26 \mathrm{C}$ & 40.0667 & 4.444 & 47.50 & 32.00 & 19.7525 \\
\hline $26 \mathrm{D}$ & 75.1111 & 11.867 & 85.50 & 50.00 & 140.8211 \\
\hline $26 \mathrm{~F}$ & 51.3222 & 5.309 & 58.70 & 41.70 & 28.1819 \\
\hline $27 \mathrm{~A}$ & 39.0222 & 2.488 & 42.00 & 35.20 & 6.1894 \\
\hline $27 \mathrm{~B}$ & 43.5889 & 5.968 & 50.50 & 34.50 & 36.6211 \\
\hline $28 \mathrm{~A}$ & 54.1444 & 3.574 & 60.60 & 51.00 & 12.7703 \\
\hline $28 \mathrm{~B}$ & 65.1778 & 3.339 & 69.50 & 57.50 & 11.1519 \\
\hline $28 \mathrm{C}$ & 69.2778 & 3.585 & 74.50 & 65.20 & 12.8544 \\
\hline $28 \mathrm{D}$ & 59.7444 & 6.335 & 67.40 & 49.50 & 40.1378 \\
\hline $28 \mathrm{E}$ & 69.4222 & 6.249 & 79.00 & 50.50 & 39.0544 \\
\hline 29 & 43.2000 & 11.492 & 52.00 & 21.00 & 132.0600 \\
\hline $30 \mathrm{~A}$ & 54.8000 & 6.317 & 62.00 & 46.50 & 39.9100 \\
\hline $30 \mathrm{~B}$ & 39.7889 & 6.643 & 53.50 & 32.50 & 44.1286 \\
\hline $31 \mathrm{~A}$ & 37.6556 & 5.963 & 49.30 & 30.50 & 35.5528 \\
\hline $31 \mathrm{~B}$ & 48.8889 & 7.875 & 58.70 & 37.50 & 62.0136 \\
\hline $32 \mathrm{~A}$ & 41.2222 & 7.082 & 48.20 & 29.50 & 50.1519 \\
\hline $32 \mathrm{~B}$ & 51.4111 & 6.331 & 58.10 & 40.50 & 40.0861 \\
\hline $32 \mathrm{C}$ & 46.3556 & 12.600 & 62.30 & 31.00 & 158.7603 \\
\hline $33 \mathrm{~A}$ & 45.4333 & 2.363 & 48.20 & 42.00 & 5.5825 \\
\hline $33 \mathrm{~B}$ & 9.5556 & 16.576 & 42.00 & 1.00 & 273.7778 \\
\hline 34 & 34.7778 & 13.236 & 42.30 & 1.00 & 175.1944 \\
\hline 35 & 27.7667 & 9.301 & 41.10 & 10.00 & 86.5150 \\
\hline 36 & 50.6889 & 5.599 & 59.10 & 44.30 & 31.3461 \\
\hline $37 \mathrm{~A}$ & 49.2889 & 7.267 & 61.40 & 39.50 & 52.8136 \\
\hline $37 \mathrm{C}$ & 59.2222 & 4.734 & 69.20 & 51.40 & 22.4144 \\
\hline $37 \mathrm{D}$ & 68.8778 & 8.285 & 79.70 & 56.50 & 68.6494 \\
\hline General average & 50.1830 & & & & \\
\hline
\end{tabular}


Table 5 - Descriptive statistics for the qualitative characteristics LLEN- leaf length (cm) and LWID- leaf width (cm) analyzed in $P$. nicorae.

\begin{tabular}{|c|c|c|c|c|c|}
\hline \multirow{2}{*}{ Accession } & \multicolumn{2}{|c|}{ LLEN } & \multicolumn{2}{|c|}{ LWID } & \multirow{2}{*}{ Groups $^{\mathrm{a}}$} \\
\hline & Average & Standard deviation & Average & Standard deviation & \\
\hline 2 & 13.0625 & 4.2592 & 0.4344 & 0.1044 & 3 \\
\hline $2 \mathrm{~A}$ & 25.3500 & 9.2433 & 0.5021 & 0.1344 & 1 \\
\hline 3 & 22.3682 & 9.1689 & 0.4941 & 0.1159 & 2 \\
\hline 4 & 34.2350 & 11.2898 & 0.5650 & 0.0587 & 1 \\
\hline $4 \mathrm{~A}$ & 28.7900 & 10.8254 & 0.5760 & 0.1033 & 1 \\
\hline 5 & 28.0478 & 6.9124 & 0.3913 & 0.0417 & 2 \\
\hline $6 \mathrm{~A}$ & 28.1143 & 10.4293 & 0.4633 & 0.0896 & 2 \\
\hline $6 \mathrm{~B}$ & 24.9944 & 10.4456 & 0.5111 & 0.1008 & 1 \\
\hline $8 \mathrm{~A}$ & 23.4250 & 11.5406 & 0.4417 & 0.0776 & 2 \\
\hline $8 \mathrm{~B}$ & 22.1917 & 8.9906 & 0.3613 & 0.0600 & 2 \\
\hline 9 & 28.4667 & 11.7201 & 0.6214 & 0.0902 & 1 \\
\hline $9 \mathrm{~A}$ & 34.0762 & 10.4466 & 0.4748 & 0.0999 & 2 \\
\hline 10 & 28.4609 & 10.2574 & 0.5000 & 0.0889 & 1 \\
\hline $11 \mathrm{~A}$ & 32.8957 & 10.6601 & 0.5783 & 0.0850 & 1 \\
\hline 11B & 28.1833 & 7.8135 & 0.6222 & 0.0808 & 1 \\
\hline $12 \mathrm{~A}$ & 35.3783 & 14.6889 & 0.6661 & 0.1398 & 1 \\
\hline $12 \mathrm{~B}$ & 35.9208 & 11.0952 & 0.6292 & 0.0806 & 1 \\
\hline $13 \mathrm{~A}$ & 26.5217 & 9.1528 & 0.5391 & 0.0783 & 1 \\
\hline 13B & 27.3000 & 9.0990 & 0.4955 & 0.0999 & 2 \\
\hline 14 & 20.9267 & 9.9136 & 0.3600 & 0.0632 & 2 \\
\hline $14 \mathrm{~A}$ & 21.5714 & 8.6756 & 0.3693 & 0.0783 & 2 \\
\hline 17 & 27.8478 & 7.0653 & 0.5413 & 0.0577 & 1 \\
\hline 18 & 23.9000 & 9.6335 & 0.4870 & 0.0626 & 2 \\
\hline 19 & 28.3296 & 10.9558 & 0.6148 & 0.0818 & 1 \\
\hline $20 \mathrm{~B}$ & 31.0739 & 7.5965 & 0.5413 & 0.0577 & 1 \\
\hline $20 \mathrm{C}$ & 25.1957 & 9.2135 & 0.6000 & 0.0798 & 1 \\
\hline $26 \mathrm{~A}$ & 28.4091 & 13.7346 & 0.4341 & 0.1357 & 2 \\
\hline $26 \mathrm{C}$ & 26.0150 & 7.6288 & 0.3800 & 0.0768 & 2 \\
\hline $26 \mathrm{D}$ & 36.2682 & 14.2699 & 0.5614 & 0.1154 & 1 \\
\hline $26 \mathrm{~F}$ & 26.0273 & 12.8726 & 0.4682 & 0.0933 & 2 \\
\hline $27 \mathrm{~A}$ & 20.2154 & 5.8609 & 0.3725 & 0.1017 & 2 \\
\hline $27 \mathrm{~B}$ & 25.9682 & 6.7062 & 0.4500 & 0.0617 & 2 \\
\hline $28 \mathrm{~A}$ & 23.4636 & 6.9416 & 0.4555 & 0.0875 & 2 \\
\hline $28 \mathrm{~B}$ & 29.2103 & 10.7882 & 0.4845 & 0.2000 & 2 \\
\hline $28 \mathrm{C}$ & 36.1333 & 8.4549 & 0.5604 & 0.0872 & 1 \\
\hline $28 \mathrm{D}$ & 29.2091 & 8.0933 & 0.4032 & 0.0752 & 2 \\
\hline $28 \mathrm{E}$ & 34.5958 & 13.2368 & 0.4750 & 0.0608 & 2 \\
\hline 29 & 24.1579 & 11.7887 & 0.4968 & 0.1279 & 2 \\
\hline $30 \mathrm{~A}$ & 32.2840 & 11.2091 & 0.5980 & 0.1005 & 1 \\
\hline $30 \mathrm{~B}$ & 29.5700 & 10.9538 & 0.5500 & 0.1179 & 1 \\
\hline $31 \mathrm{~A}$ & 24.5722 & 4.7128 & 0.5300 & 0.1261 & 1 \\
\hline $31 \mathrm{~B}$ & 23.3550 & 8.6878 & 0.4450 & 0.0605 & 2 \\
\hline $32 \mathrm{~A}$ & 21.2421 & 6.5709 & 0.4947 & 0.0848 & 2 \\
\hline $32 \mathrm{~B}$ & 23.7500 & 7.9316 & 0.5050 & 0.0999 & 1 \\
\hline $32 \mathrm{C}$ & 28.9875 & 10.7668 & 0.5013 & 0.0981 & 1 \\
\hline 34 & 22.1231 & 8.5608 & 0.6731 & 0.1194 & 1 \\
\hline 35 & 14.6556 & 5.8520 & 0.4333 & 0.1283 & 3 \\
\hline 36 & 28.6917 & 8.0031 & 0.5008 & 0.0770 & 1 \\
\hline $37 \mathrm{C}$ & 27.8765 & 11.6050 & 0.4800 & 0.0752 & 2 \\
\hline $37 \mathrm{D}$ & 30.8077 & 7.0276 & 0.4592 & 0.0511 & 2 \\
\hline General average & 26.9139 & & 0.5302 & & \\
\hline
\end{tabular}

${ }^{a}$ Groups 1- long (more than $20 \mathrm{~cm}$ ) and wide (more than $0.5 \mathrm{~cm}$ ) leaves; 2- long (more than $20 \mathrm{~cm}$ ) and narrow (smaller than $0.5 \mathrm{~cm}$ ) leaves; 3 - and the small (less than $20 \mathrm{~cm}$ ) and narrow (smaller than $0.5 \mathrm{~cm}$ ) leaves. 


\section{Conclusions}

There is a high morphological variation for all characteristics analyzed among the $P$. nicorae accessions studied, but is not related to their geographical location.

This richness of variation represents an extremely valuable source to select better accessions to be used as forage plants.

\section{Acknowledgements}

To Conselho Nacional de Desenvolvimento Científico e Tecnológico (CNPq, Brazil), Coordenação de Aperfeiçoamento de Pessoal de Nível Superior (CAPES, Brazil) and Fundação de Amparo à Pesquisa do Estado do Rio Grande do Sul (FAPERGS, Brazil), for financial support and grants, Dr. Ilsi Iob Boldrini for taxonomic identification of the plants and MSc. Danilo Menezes Sant'Anna for help in the collections.

\section{References}

Asker, S.E.; Jerling, L. 1982. Apomixis in plants. CRC Press, Boca Raton, FL, USA.

Barreto, I.L. 1974. O gênero Paspalum (Gramineae) no Rio Grande do Sul. UFRGS, Porto Alegre, RS, Brazil.

Boldrini, I.I. 2006. Biodiversidade dos campos sulinos. p. 11-24. In: Dall'Agnol, M.; Nabinger, C.; Rosa, L.M.; Silva, J.L.S.; Santos, D.T.; Santos, R.J., eds. Anais do I Simpósio de Forrageiras e Produção Animal. Faculdade de Agronomia, UFRGS, Porto Alegre, RS, Brasil.

Boldrini, I.I.; Longhi-Wagner, H.M.; Boechat, S.C. 2005. Morfologia e taxonomia de gramíneas sul-riograndenses. UFRGS, Porto Alegre, RS, Brasil.

Botelho, M.R.; Dalmolin, R.S.D.; Pedron, F.A.; Azevedo, A.C.; Rodrigues, R.B.; Miguel, P. 2006. Color measurement in soils from Rio Grande do Sul State with Munsell charts and by colorimetry. Ciência Rural 36: 1179-1185 (in Portuguese, with abstract in English).

Burkart, A. 1969. Flora ilustrada de Entre Rios-Gramineae. INTA, Buenos Aires, Argentina.

Burson, B.L.; Bennett, H.W. 1970. Cytology, method of reproduction, and fertility of Brunswickgrass, Paspalum nicorae Parodi. Crop Science 10: 184 -187.

Callegaro, J.M.; Pezzi, E.; Bender, R.J. 2002. Use of modified atmosphere storage on postharvest quality of strawberries. Pesquisa Agropecuária Brasileira 37: 1049-1055 (in Portuguese, with abstract in English).
Dahmer, N.; Schifino-Wittmann, M.T.; Dall'Agnol, M.; Castro, B. 2008. Cytogenetic data for Paspalum notatum Flügge accessions. Scientia Agricola 65: 381-388.

Dall’Agnol, M.; Steiner, M.G.; Baréa, K.; Scheffer-Basso, S.M. 2006. Perspectivas de lançamento de cultivares de espécies forrageiras nativas: o gênero Paspalum. p.149-162. In: Dall'Agnol, M.; Nabinger, C.; Rosa, L.M.; Silva, J.L.S.; Santos, D.T.; Santos, R.J., eds. 2006. Anais do I Simpósio de Forrageiras e Produção Animal. Faculdade de Agronomia, UFRGS, Porto Alegre, RS, Brasil.

Moraes-Fernandes, M.I.B.; Barreto, I.L.; Salzano, F.M. 1968. Cytogenetic, ecologic and morphologic studies in Brazilian forms of Paspalum dilatatum. Canadian Journal of Genetics and Cytology 10:131-138.

Moraes-Fernandes, M.I.B.; Barreto, I.L., Salzano, F.M.; Sacchet; A.M.O.F. 1974. Cytological and evolutionary relationships in Brazilian forms of Paspalum (Gramineae). Caryologia 27: 455-465.

Nabinger, C.; Dall'Agnol, M. 2008. Principais gramíneas nativas do RS: características gerais, distribuição e potencial forrageiro.p. 7-54. In: Dall'Agnol, M.; Nabinger, C.; Santos, R.J., eds. 2008. Anais do III Simpósio de Forrageiras e Produção Animal. Faculdade de Agronomia, UFRGS, Porto Alegre, RS, Brasil.

Pagliarini, M.S.; Carraro, L.R.; Freitas, P.M.; Adamowski, E.V. Batista, L.A.R.; Valls, J. F. M. 2001. Cytogenetic characterization of Brazilian Paspalum accessions. Hereditas 135: 27-34.

Pedreira, C.G.S.; Pedreira, B.C. 2006. A grama-forquilha (Paspalum notatum). p. 115-148. In: Dall'Agnol, M.; Nabinger, C.; Rosa, L.M.; Silva, J.L.S.; Santos, D.T.; Santos, R.J., eds. 2006. Anais do I Simpósio de Forrageiras e Produção Animal. Faculdade de Agronomia, UFRGS, Porto Alegre, RS, Brasil.

Pizzarro, E.A. 2000. Potencial forrajero del género Paspalum. Pasturas Tropicales 22: 38-46.

Prestes, P.J.Q.; Freitas, E.A.G.; Barreto, I.L. 1976. Hábito vegetativo e variação estacional do valor nutritivo das principais gramíneas da pastagem nativa do Rio Grande do Sul. Anuário Técnico do Instituto de Pesquisas Zootécnicas "Francisco Osório" 3: 516-531.

Quarín, C.L.; Norrmann, G.A. 1990. Interspecific hybrids between five Paspalum species. Botanical Gazette 151: 366-369.

Reis, C.A.O.; Schifino-Wittmann, M.T.; Dall'Agnol, M. 2008. Cytogenetic characterization of a collection of Paspalum nicorae Parodi accessions. Crop Breeding and Applied Biotechnology 8: 212-218.

Valls, J.F.M. 2000. Impacto do conhecimento citogenético na taxonomia de Paspalum e Axonopus (Gramineae). p.15-21.In: Cavalcanti, T.B.; Walter, B.M.T., eds. Tópicos atuais em botânica. Embrapa-Cenargen, Brasília, DF, Brasil.

Received May 08, 2009

Accepted December 21, 2009 